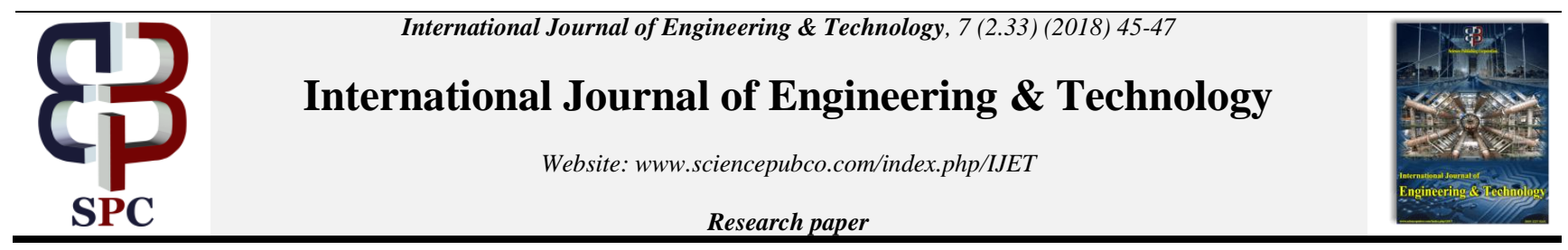

\title{
A rational approach in the sensory stimuli enhancement for the agnosic
}

\author{
Ms. S. Anitha Jebamani ${ }^{1}{ }^{*}$, Ms. R. Divya ${ }^{2}$, Ms. K. Smruthi ${ }^{2}$ \\ ${ }^{1}$ Asst.Prof ,Department of Information Technology, Sri Sai Ram Engineering College \\ ${ }^{2}$ Department of Information Technology, Sri Sai Ram Engineering College \\ *Corresponding author E-mail: jebamani.it@sairam.edu.in
}

\begin{abstract}
Life in today's world has become very competitive and challenging. In this ambitious realm, learning, perception, application and expression are vital to every existential being. However, there are a few people around the world who are bereft of these fundamentals of liveli-hood. Agnosia is a disorder that exhibits the diminution of the ability to recognize objects, sounds or other sensory stimuli. It Is sometimes described as 'perception without meaning'. The victim still has the knowledge of the object or the sound, but is unable to asso-ciate it meaningfully. There are varied dimensions of this disorder including visual, auditory and color agnosia.

The aim of our project is to create a mobile application for the subjects of this disorder to be able to improve their recognition of objects, faces, sounds and colors through persistent practice and analysis. Illustrations of persons and objects with their descriptions for visual recognition, along with a verbal guide for identification and perception of sounds, and a color-picker to identify right colors will be equipped for study. Assessment criteria for evaluation of their performance will also be devised to keep in check their improvement, based on which graphical overviews and reports will be generated. A pulse sensor will also be appended to keep the pulse of the victim in check while on assessment. This methodical approach could be efficacious for the progression of sensory perception and recognition in the agno-sic.
\end{abstract}

Keywords: Disorder; Recognition; Perception Without Meaning; Visual; Auditory; Pulse Sensor; Assessment.

\section{Introduction}

Agnosia is a perceptual disorder in which sensation is preserved but the ability to recognize a stimulus or know its meaning is lost. Agnosia means "without knowledge". Patients with agnosia cannot understand or recognize what they see, hear or feel.

When assessing agnosia, it is important to establish that sensation is preserved; the patient is alert, intelligence is intact (or near intact) with no language or memory disorder. Examination involves assessing what the patient sees, hears or feels when presented with objects, pictures or sounds using a combination of clinical procedures and neuropsychological tests. [1].

In regards to this affliction, we are introducing a rational approach for addressing difficulties faced by sensory stimuli to respond to normal entities - a mobile application for truncating the effects of Agnosia through persistent exposure and tuition.

\section{Existing system}

There is no actual cure for this disease called Agnosia.

Neither does it have a proper treatment which can be completely relied on. Regular and continuous inputs are given to the victim until it is recognized and sinked in.

Currently, there is no mobile or web application for treating Agnosia. Agnosic people are generally sent to Rehabilitation centre and they undergo incessant training. [1] They are given occupational therapy and speech therapy which leads them to improve over a period of time. ${ }^{[5]}$ These training sessions has to be methodi- cal and consistent so that some effect is seen in the people with disorder.

- The main disadvantage of the existing system is that a sole resource is needed to attend to Agnosic patients.

- Time, resource and cost is high.

- Each and every person is not necessarily given the same and individual care.

\section{Proposed system}

The objective of our project is to introduce a mobile application for the agnosic persons to be able to conveniently and efficiently alleviate their curtailment of prompt recognition and perception. [2] The mobile application is built with the following aspects: predefined illustrations of common objects and personalities, whose descriptions will also be appended for better understanding and easier instilling to stimuli; figurative sounds and pronunciation of the object or face to address impairment of perception while hearing; an intuitive approach for proper color recognition using colorpicker; a server-supported database for storing the user-added additional objects and faces; a module for conducting prescribed tests based on doctor-chosen parameters of examination; and cumulative reports, summary and charts for comprehensive evaluation of individual performance and improvement in the subject's condition. An option to translate the existing word into the required language is also fitted for convenient comprehension. In addition to the in-built app features, a pulse sensor is also attached as an add-on to detect the pulse rate of the victim while undergo- 
ing the examination, which if not necessary could be subjugated and detached.

On studying the effects of this disorder, many case reports show improvement following intensive rehabilitation. The core principles of treatment are restitution and repetitive training of impaired function. This could greatly be dealt with by utilizing this mobile application for constant exposure and focus over any point of time. Considering that mobile phones are portable and this application does not require the adjunct of Internet connection, it can be used anywhere any time. [2] Ultimately, our mobile application will prove to be efficacious for the progression of sensory perception and recognition in the agnosic.

\section{Architecture diagram}

The architecture for the proposed system consists of a presentation layer,application layer and the database layer as below.

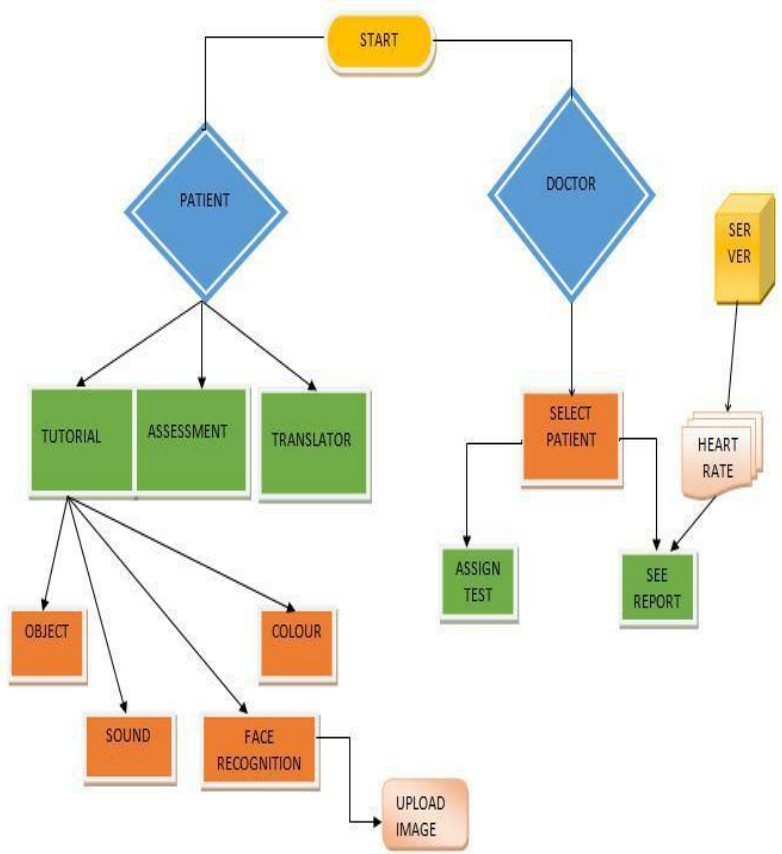

Fig. 1: Overall Architecture.

The presentation layer consists of the authentication credentials for both the patient and the doctor.It consists of verbal guide,auditory guide,object and colour recognition guide ,assessments and translation of basic words into different languages.The patient has to choose upon which guide they need. The application layer will have a detailed description of the objects,faces and sounds.Activities such as tests are taken by the patient and the questions are set up by doctors in this very layer. Finally, the database layer constitutes of all the predefined data such as the illustrations and descriptions of the objects and the audio files for auditory recognition and the results of the tests will be stored.

\section{Work flow}

The workflow of the proposed system will be as below

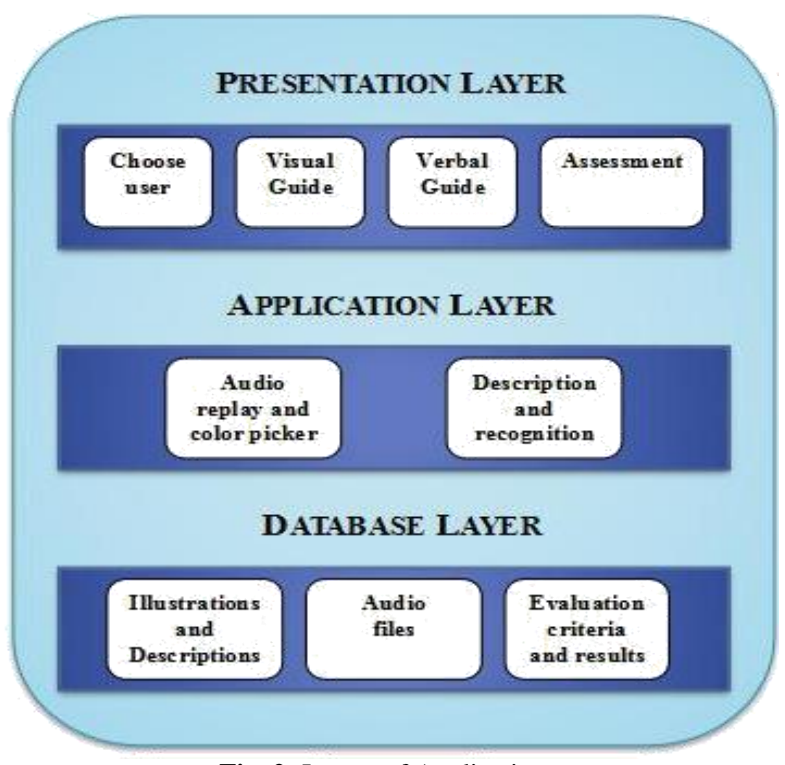

Fig. 2: Layers of Application.

The application will have a separate login for the patient and the doctor. Here the patient will act as a user and the doctor will act as a admin.Both the former and the latter will have registration and login, wherein the patient can register themselves under the doctor they wish to.

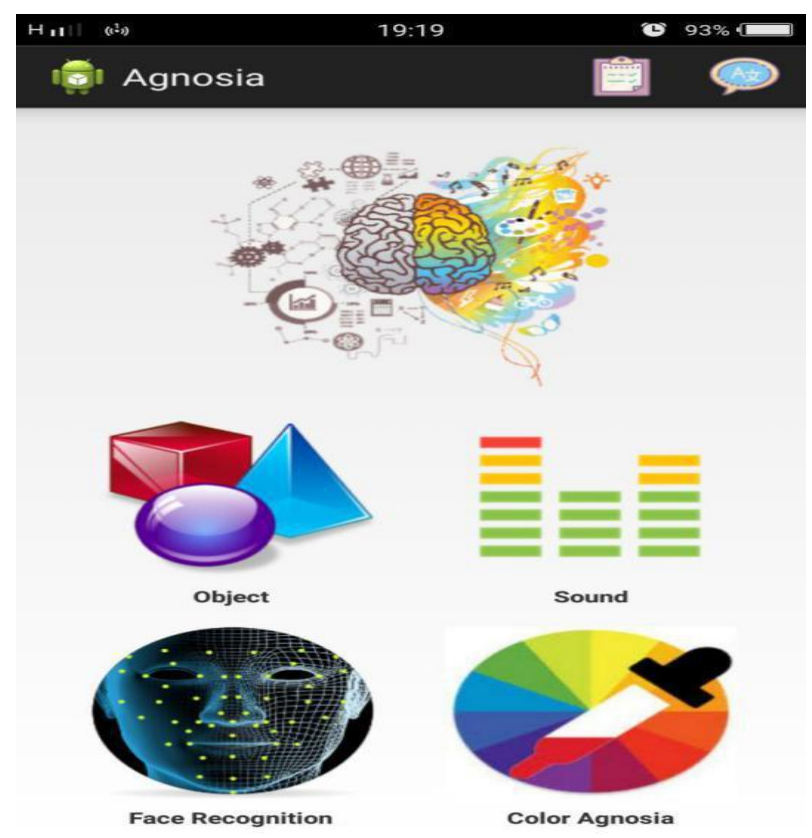

Fig. 3: UI of Agnosia

The patient will be provided with tutorials,assessments and translators. The tutorials will have illustrations and descriptions of the objects ,sounds and faces.Audio files will also be included to enhance the auditory recognition.As each patient will will across varying kinds of people in their lifetime, an option to upload the picture and description about the person has been provided.The patients can take assessments only when doctor under whom they have registered schedule them the assessments.An additional option of translation has been included with which the person can translate and practise the basic words in different languages.

The doctor after logging in has to select the patient under him.After which has will be provided with two options, one he can set the assessment question for that particular patient and,the other option is he can see the test reports of the patient such as their score.

As the patients with agnosic kind of disorder will have abnormal and varying kind of pulse rates, pulse sensor will be incorporated 
and the patients pulse rates can be monitored through a separate application in the form of graph.

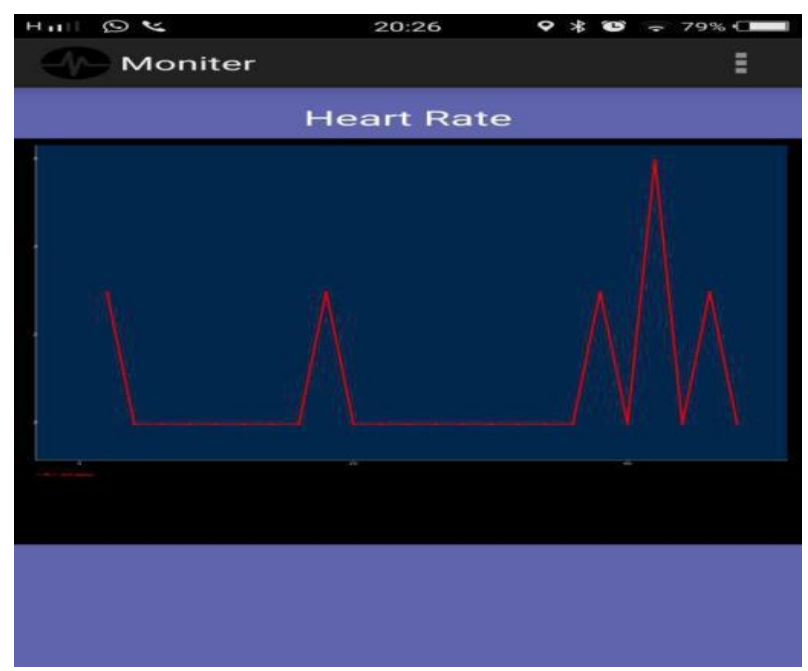

Fig. 4: Monitoring System.

\section{Future prospects}

More in-depth concentration towards Auditory Agnosia could be rendered by adding non-linguistic sound tests, training towards spoken language for cases with pure word deafness and instilling the ability to reproduce a familiar musical note by listening and humming or singing. [3] [4] Separate modules for coping with pure word blindness by including options to write a dictated phrase or read a written stanza could be implemented. Animated objects, graphics or tactile games could be inculcated in the application to make it more attractive, lively and relatable for the victim.

\section{Conclusion}

Thus, the application will provide a residential guidance and provide a convenient therapeutic measure for compensation of defects and also provides a facility to track improvement through tests.

\section{References}

[1] Matthew Velazquez , Yugyunglee Inte National Conference Onhealthcare Informatics (Ichi)-Cognitive Visual Recognition Tracker

[2] Journal Of Cognitive Neuroscience- Volume 4, Issue 2-Preserved Visual Imagery And Categorization In A Case Of Associative Visual Agnosia- Janet Jankowiak , Marcel Kinsbourne , Ruth S.Shalev , David L. Bachman

[3] An Examination Of Visual Agnosia-Candace N.Palmer-Stephen F.Austin State University Spring 2002 